\title{
UNA LECTURA PERSPECTIVISTA DE LA NEUROFENOMENOLOGÍA: Francisco VARELA Y RONALD GIERE
}

\author{
A Perspectivist Reading of Neurophenomenology: \\ FRANCISCO VARELA AND Ronald Giere
}

\author{
Ricardo Mejía Fernández \\ Universidad de Salamanca (Salamanca, España) \\ Université Catholique de Louvain (Louvain-la-Neuve, Bélgica) \\ ricardomejia@usal.es
}

\begin{abstract}
Resumen: El autor realiza una lectura en clave perspectivista de la neurofenomenología, enfoque transdisciplinar y metodológico iniciado a mediados de los 90 por el neurobiólogo Francisco J. Varela desde el ámbito experimental de las ciencias de la mente. La originalidad del artículo reside en que estudia comparativamente el problemático entrecruce de la neuro-fenomenología de Varela con el perspectivismo científico de Ronald N. Giere, mostrándonos que la neurofenomenología no sólo lo antecedió cronológicamente, sino que fue mucho más radical al te-ner en cuenta la la dimensión fenomenológica y trascendental de la experiencia vivida humana y al aplicarla en los protocolos de las neurociencias cognitivas.
\end{abstract}

Palabras clave: Perspectivismo, Neurofenomenología, Varela, Fenomenología.

\begin{abstract}
The author makes a perspectivist reading of neurophenomenology, a transdisci-plinary and methodological approach pioneered in the 90 's by the neurobiologist Francisco J. Varela, who worked in the experimental field of the science of mind. The originality of the article consists in studying with a comparative method the problematic intertwine of Varela's neuro-phenomenology and the scientific perspectivism recently defended by Ronald N. Giere. In this way, the autor shows how neurophenomenology not only came before Giere's proposal but also how it was more radical in considering the phenomenological and transcendental human dimension and in trying an implementation in cognitive neuroscience protocols
\end{abstract}

Keywords: Perspectivism, Neurophenomenology, Varela, Phenomenology.

\section{INTRODUCCIÓN}

El objetivo de este artículo es llevar a cabo una lectura en clave perspectivista de la neurofenomenología, enfoque transdisciplinar y metodológico iniciado por el neurobiólogo Francisco J. Varela (1946-2001) en el propio seno de la 
neurociencia cognitiva. Se tratará, pues, de emprender una investigación original no en el sentido de que resaltemos el perspectivismo exclusivamente de la neurofenomenología (una tesis conocida), sino en que es la primera vez en que se verá el entrecruce problemático y no concordista de la neurofenomenología vareliana con el perspectivismo científico de Ronald N. Giere (1938-). Como es sabido, esta corriente es muy relevante en la actual filosofía de la ciencia al contar con autores afines de la importancia de Van Fraassen ${ }^{1}$, Rueger ${ }^{2}$ o Morrison ${ }^{3}$.

Nuestra originalidad, insistimos, es poner en contacto a dos autores muy diferentes: uno propio de la tradición post-empirista y otro del enactivismo en neurociencias. Si bien no podemos negar que no hay actualmente ninguna publicación sobre el perspectivismo stricto sensu en Varela, lo que perseguimos, por tanto, no es hacer que ambos concuerden artificialmente en todo sino destacar la base común que comparten (y difieren) de cara a integrar lo perspectivizado en el perspectivizador a través de aquellos principios y contenidos (imposible en todos) de sus respectivos pensamientos. A la vez que vinculamos a Francisco $\mathrm{J}$. Varela con la tradición perspectivista in genere lo relacionamos con un autor perspectivista en concreto (Ronald N. Giere), el cual se autodenomina de ese modo. Esta es la peculiaridad de nuestro artículo, así como su problematicidad. Así pues y tras la presente introducción (1), abordaremos el perspectivismo común en sus inicios históricos (2), para más tarde detenernos en la propuesta de Giere en su conexión con la vareliana (3) y, en el penúltimo punto (4), ocuparnos de la compleja relación del perspectivismo científico gieriano y la neurofenomenología vareliana. Finalmente (5), aportaremos unas resumidas conclusiones.

\section{EL PERSPECTIVISMO Y SUS INICIOS}

La neurofenomenología fue introducida por Varela a mediados de los años 90 mientras era director de investigación del laboratorio de neurociencias del Centro Nacional de Investigación Científica de París (CNRS, en sus siglas en francés). Esta es definida como un remedio metodológico (methodological remedy) del

\footnotetext{
${ }^{1}$ Cfr. Van Frassen, Bas. Scientific representation. Paradoxes of perspective, Nueva York: OUP, 2008.

2 Cfr. Rueger, Alexander. "Perspectival models and theory unification", British Journal for the Philosophy of Science, No LVI, 2005.

${ }^{3}$ Cfr. Morrison, Margaret. "One phenomenon, many models: Inconsistency and complementarity", Studies in History and Philosophy of Science, No XLII, 2011.
} 
problema de la conciencia (o mejor de su vacío explicativo) que nos hace cuestionarnos si es, de algún modo, una variante del perspectivismo. Así las cosas, el acento más alto lo recibirá el punto de vista del sujeto, denominado también "perspectiva" en la historia del pensamiento. Este punto de vista de la primera persona, al que no le repugna el de la tercera persona sino todo lo contario, es lo que se ha llamado "perspectiva" explícitamente (en Ortega y Nietzsche) e implícitamente (en Leibniz o en el propio Varela).

Para empezar, bastará con exponer y argumentar, más allá de lo semántico, los grandes trazos históricos de este movimiento. Como siempre, el perspectivismo no es un tótem robusto al que haya que postrarse, sino que, por el contrario, es preciso sostener que, en su generalidad, mantiene la aserción de que todo conocimiento, para que adquiera la cualidad de "conocido", ha de ser perspectivo para un sujeto o comunidad de sujetos.

En Verdad y perspectiva, Ortega y Gasset aseveraba, a favor de esta aserción básica de perspectivismo que "el punto de vista individual me parece el único punto de vista desde el cual puede mirarse el mundo en su verdad. Otra cosa es un artificio"4. En analogía al espectador lo que interesa no es los objetos que contempla sino "la vida según fluye ante él") ${ }^{5}$. Ciertamente, Ortega era más reticente a este carácter comunitario de la perspectiva en pro del punto de vista individual, al defender que

cada hombre tiene una misión de verdad. Donde está mi pupila no está otra: lo que de la realidad ve mi pupila, no lo ve otra. Somos insustituibles, somos necesarios. [...] La realidad, pues, se ofrece en perspectivas individuales. Lo que para uno está en último plano, se halla para otro en primer término ${ }^{6}$.

Más que de (un) perspectivismo, puesto que la perspectiva toma una forma en cada sujeto, se ha preferido hablar de (muchos) perspectivismos según la pluralidad de puntos de vista, aunque todos emiten con sus propios matices la asunción general que acabamos de definir. Si seguimos recordando a Ortega, "la realidad, precisamente por serlo y hallarse fuera de nuestras mentes

\footnotetext{
${ }^{4}$ Ortega Y Gasset, José. "Verdad y perspectiva", en Obras Completas, Madrid: Taurus, 2004, Tomo II, p. 162.

${ }^{5}$ Ibíd.

${ }^{6}$ Ibíd., p. 163.
} 
individuales, sólo puede llegar a éstas multiplicándose en múltiples caras o ha-

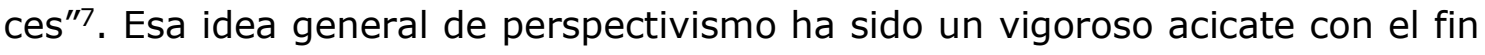
de resituar en un lugar destacado al sujeto en la tarea reflexiva y científica.

Con anterioridad a Ortega y no siendo ajeno a ello este pensador, fue Leibniz quien, en la ilustración racionalista, legó para la posteridad un decisivo perspectivismo en el que, según escribía este filósofo en sus Nuevos Ensayos, "el alma es un pequeño mundo [petit monde] donde las ideas distintas son una representación de Dios y donde las confusas son una representación del universo"8. Este pequeño mundo de cada mónada concretada en el ser humano variará, digámoslo sin vacilar, según quién represente tanto lo distinto como lo confuso. Más bien, cada alma es inigualable en esa representación en razón de que disfruta de una perspectiva singular y originaria en el universo:

[...] dos almas humanas, o dicho de otro modo, de una misma especie, no salen jamás perfectamente semejantes de las manos del Creador, y tienen siempre cada una su relación originaria [rapport originaire] a los puntos de vista [points de veu] que ellas tendrán en el universo ${ }^{9}$.

Como ilustraba Henri Lachelier, antiguo editor de Leibniz, "las mónadas perciben todas el mundo con un punto de vista diferente, y es necesario que sea establecido el acuerdo entre los puntos de vista de todas las mónadas [...] cada una de las mónadas extrae todas las percepciones de su propio fondo"10. Puesto que estas mónadas no son etéreas sino concretas en contra de todo dualismo, ha habido quien ha apreciado en Leibniz un "perspectivismo corporal" por el que, en la "unidad real" monádica, "la corporalidad no sólo es principio de orden, sino también principio de vida"11. Gracias a los puntos metafísicos (o matemáticos) como enfoque metodológico más idóneo, en "esta gnoseología corporal-perspectivista cada individuo se sitúa en relación al mundo de modo peculiar"12. Más

\footnotetext{
7 Ibíd., p. 127.

${ }^{8}$ Leibniz, Gottfried Wilhem. Nouveaux Essais sur I'Entendement. Opuscules divers, en Oeuvres de Leibniz, M.A. Jacques, ed., Lyon: Charpentier, 1842, Tomo I, p. 58.

${ }^{9}$ Ibíd., p. 11.

10 Lachelier, Henri. "Introducción", en G.W. Leibniz, Nouveaux Essais sur I'Entendement Humain, Paris: Librarie Hachette, 1898 , p. 36.

${ }^{11}$ Nicolás, Juan Antonio. "Gnoseología y perspectivismo corporal en Leibniz", Cuadernos Salmantinos de Filosofía, No V, 10, 2001, p. 150.

12 Ibíd., p. 139.
} 
recientemente y sobre esta corporalización no ha faltado quien corrobora "el carácter perspectivista de la mónada, pues ésta no representa el cuerpo y con ello el mundo de un modo figurativo"13. Leibniz bien pudiera ser tenido como uno de los antecesores del perspectivismo corporalizado de la neurofenomenología que veremos más adelante.

Ya en la contemporaneidad fue Friedrich Nietzsche quien adoptó con más vehemencia dicha idea, tal y como podemos leer en Más allá del bien y el mal, donde afirmaba que "no existiría vida alguna a no ser sobre la base de apreciaciones y de apariencias perspectivistas"14. Las variadas usanzas terminológicos perspectivistas habían iniciado con este filósofo. El perspectivismo nietzscheano "trata de llevar al límite la relación sujeto objeto y de demostrar la debilidad del entendimiento humano, desenmascarando las ilusiones que genera el conocimiento y los dogmatismos que crea"15. Es de lamentar que la ciencia empírica de comienzos del siglo pasado no supiese ver que, incluso en la actitud extrema nietzscheana, "la función que se le da al perspectivismo, por lo tanto, es la de desarraigar la creencia metafísica según la cual la subjetividad es capaz de dominar la totalidad del ser"16. Como se ve, Nietzsche lanza una dura crítica, en nombre de la primacía de la vida, a la arrogancia del sujeto al atribuirse una certeza indiscutible y no criticada, toda vez que proclamaba su papel cognoscitivo central en la ciencia.

Como Nietzsche, pero sin compartir lo extremado de su planteamiento en detrimento de los hechos objetivos, lo que busca implementar el perspectivismo actual en la ciencia es recuperar la función vital del conocimiento, es decir, que conocer no es únicamente conocer objetos, sino que esta actividad pertenece a un cognoscente, a un ser humano viviente. Esto lo encontramos muy gráficamente expresado por Nietzsche en Humano, demasiado humano:

Es verdad que podría haber un mundo metafísico; no podemos negar apenas la posibilidad absoluta de ello. Consideramos todas las cosas a través de la cabeza humana y

13 Sánchez Rodríguez, Manuel. "El perspectivismo en los Nuevos ensayos sobre el entendimiento humano de Leibniz", Dissertatio. Revista de Filosofía, Vol. Comp., No 3, 2016, p. 92.

${ }^{14}$ Nietzsche, Friedrich. Jenseits von Gut und Böse. Vorspiel einer Philosophie der Zukunft, § 34, KSA, V, p. 60, en L. de Santiago Guervós, "Las ilusiones del conocimiento: perspectivismo e interpretación", Thémata, No XXVII, 2001, p. 124.

${ }^{15}$ Ibíd. p, 124.

${ }^{16}$ Ibíd. 
no podemos cortar esta cabeza; aunque permanezca la cuestión de lo que habría aún del mundo, si nosotros la cortásemos ${ }^{17}$.

Como acabamos de leer, el perspectivismo de Nietzsche, si bien no estuvo entre sus logros extrapolarlo a la ciencia empírica, nos alerta irónicamente contra un pensamiento sin cabeza. Y no sólo en la metafísica: la ciencia empírica ha pensado sus objetos sensibles como decapitando a la subjetividad que los piensa. A esto quiere dar respuesta el perspectivismo científico que veremos en el punto siguiente.

\section{EL PERSPECTIVISMO CIENTÍFICO}

En 2006, el filósofo de la ciencia Ronald N. Giere publicó su libro Scientific perspectivism, obra en la que se dará por iniciado ostensiblemente el perspectivismo científico en la ciencia, si bien en este artículo mostraremos cómo Varela elaboró con anterioridad muchos de sus presupuestos. Es cierto que, en la historia de la ciencia, ha habido posturas perspectivistas como, por ejemplo, la de una columna de la física cuántica de la talla de Werner Heisenberg, el cual en 1971 y como bien recoge Capra, defendía la dependencia que los hechos guardaban con respecto al punto de vista del investigador: "Lo que observamos no es la naturaleza en sí misma, sino la naturaleza expuesta a nuestro método de observación"18.

Sería, pues, faltar a la verdad afirmar que antes de Varela o Giere no hubo ningún intento de recuperar el tema de la perspectiva en ciencia, lo cual, como leemos en la mencionada obra, ya se encontraba insinuado los autores que hemos visto al comenzar este artículo: "El perspectivismo tiene antecedentes en el trabajo de filósofos mucho anteriores, tales como Leibniz, Kant y Nietzsche"19. Con todo, Giere quiso introducir este perspectivismo en la ciencia de nuestros días: "Mi perspectivismo, [nos dice], está desarrollado casi mayoritariamente dentro del marco de la ciencia contemporánea"20. Este autor tuvo el denuedo de incorporar el perspectivismo científico no sólo como concepto sino como un modo

\footnotetext{
17 Nietzsche, Friedrich, Menschliches, Allzumenschliches I, § 9, KSA, I, p. 29, en L. de Santiago Guervós, "Las ilusiones del conocimiento: perspectivismo e interpretación", Thémata, No XXVII, 2001, p. 125.

${ }^{18}$ Capra, Fritjof, La trama de la vida, Barcelona: Anagrama, 1996, p. 60.

${ }_{19}$ Giere, Ronald N. Scientific perspectivism, Chicago: The University of Chicago Press, 2006, p. 3.

${ }^{20}$ Ibíd.
} 
muy original de pensar la ciencia. La naturaleza del conocimiento científico no es absoluta ni neutral ya que viene influenciada por la práctica y perspectivas de agentes humanos.

\subsection{Atenuación de los antagonismos en la ciencia}

Antes de la obra que comentamos, Giere ya se había referido en los años 90 a un "perspectivismo realista" o a un "realismo constructivo"21, aunque sin ofrecer una articulación final en su relación con la ciencia. De hecho, la finalidad del perspectivismo científico gieriano la expresa muy sucintamente en Scientific perspectivism: "Es desarrollar una comprensión de las demandas científicas que medie entre un objetivismo fuerte de muchos científicos, o el realismo fuerte (hard realism) de muchos filósofos de la ciencia, y el constructivismo encontrado ampliamente entre los historiadores y los sociólogos de la ciencia"22. La finalidad consiste, entonces, en mitigar conflictos entre los filósofos realistas de la ciencia (algunos partidarios de un realismo fuerte) y los constructivistas, atemperando de ese modo los radicalismos de cada una de las partes. No todo es rechazable en realistas ni en constructivistas, sino que hay que hacer justicia a los elementos positivos en sus reflexiones, aunque "evitando los excesos"23.

Para Giere, el exceso más impertinente es, por un lado, el realismo metafísico ("metaphysical realism"24), al igual que lo calificaba Putnam²5, y, por otro lado, el relativismo alocado ("silly relativism"26). El primero consiste, al tomar Giere la definición de Putnam, en una descripción del mundo que se tiene por completa y verdadera, o, parafraseando al Nobel Steven Weinberg, "el trabajo de la ciencia es precisamente el sentido de que hay verdades ahí fuera (out there) para ser descubiertas, verdades que una vez descubiertas formarán una parte permanente del conocimiento humano"27. En esta versión desmedida del realismo, la verdad reside ahí fuera (out there), como algo en lo que no interviene primariamente la actividad cognoscitiva humana ya que, más bien, dicha actividad surge después del descubrimiento de los objetos. Es decir, en esta versión del realismo

\footnotetext{
${ }^{21}$ Cfr. Giere, Ronald N., Science without laws, Chicago: The University of Chicago Press, 1999.

${ }^{22}$ Giere, Ronald N., Scientific perspectivism, op. cit., p. 3.

${ }^{23}$ Ibíd.

${ }^{24}$ Ibíd., p. 4.

25 Cfr. Putnam, Hilary, Reason, Truth, History, Cambridge: Cambridge University Press, 1981.

${ }^{26}$ Giere, R. N. Scientific perspectivism, op. cit., p. 13.

27 Ibíd., p. 4.
} 
no hay ni siquiera una versión clásica de la verdad como adequatio intellectus et rei, sino que la verdad sería independiente ahí fuera, de la cual el sujeto sería pasivo y no tendría más remedio que captar. Y aún más: la verdad, en el caso de las leyes físicas, pertenece a estas mismas leyes físicas sin hacer justicia a la subjetividad, la cual no sólo descubre las leyes, sino que formula dichas leyes y las transforma en teorías o las modeliza matemáticamente.

Giere admite que no todo realismo es tan inmoderado como el metafísico, sino que, aunque insuficiente, puede hallarse un "realismo objetivo" (objective realism) no claramente posicionado en contra del rol constructivo del conocimiento, por lo que podría ser encauzado hacia una devolución del lugar insustituible de la subjetividad en la caracterización de los hechos objetivos conocidos. Este "realismo objetivo" puede mejorarse con un "realismo perspectivo" (perspectival realism): "Me gustaría rechazar el realismo objetivo (objective realism) pero mantener aún un tipo de realismo, un realismo perspectivo (perspectival realism), el creo que caracteriza mejor al realismo en ciencia"28. Esto significa que el realismo perspectivo pule el método científico. La ciencia empírica, al contrario de lo que el realismo espera, no aporta "una completa y literalmente correcta imagen del mundo en sí mismo"29, ya que esta ciencia está hecha por científicos y comunidades científicas, es decir, seres humanos situados en una sociedad y comunidad política determinadas; que son integrantes de una historia. Esto significa que toda imagen del mundo es perspectiva para los sujetos, por lo que de la calidad del análisis de esta perspectiva se podrá saber más o menos acerca de dicha imagen y de las razones que han llevado a su construcción. Hay una tarea purificadora.

Así, el perspectivismo científico de Giere no adolece de un relativismo cultural porque la cultura no acaba con el mínimum de realidad a la que todos accedemos. Más bien, la realidad, sin por ello desaparecer, está culturalizada desde el inicio. Giere asume, pues, un realismo básico en el que se estudia no sólo lo construido por la ciencia sino la construcción misma de la realidad: la actividad de la ciencia es, por tanto, una dinámica que concierne a una única realidad, como única es la naturaleza que se perspectiviza. Es preferible decir "perspectivizar" que

\footnotetext{
${ }^{28}$ Ibíd., p. 5.

${ }^{29}$ Ibíd., p. 6.
} 
"construir" ya que la acción que da lugar a las perspectivas remite, en todas ellas, a una única realidad natural, mientras que "construir" tiene la connotación de crear lo que no es real, de poner en la realidad natural lo que previamente no forma parte de ella. Es necesario, como nos pide Giere, reconocer que el trabajo científico puede ser "en parte (in part) socialmente construido, y así para la posibilidad de descubrir las contribuciones sociales para estas demandas"30.

Este constructivismo mitigado en parte (in part), como afirma el autor es reelaborado como un perspectivismo donde la objetividad recibe una impronta socio-histórica desde el punto de vista de los científicos, pero sin perder su cualidad objetiva en el quicio de la perspectiva. Tenemos que aclarar, antes de proseguir, que constructivismo no equivale sin más a perspectivismo ya que se puede ser perspectivista sin ser constructivista (no así al revés). Varela y Giere son un ejemplo de ello porque la perspectiva es impracticable sin el componente sensorial y motor del cuerpo. Lo mismo vale para la relación constructivismorealismo: puede haber un constructivismo medianamente realista et viceversa (este es el fin de la propuesta científica gieriana).

\subsection{Perspectivismo científico y neurofenomenología como "via media"}

No hemos de pensar en un dilema irresoluble entre realismo metafísico o constructivismo radical porque es justamente (y solamente) en la perspectiva natural donde confluyen, epocalmente y corporalizadamente, lo perspectivizado y la perspectivización. La perspectiva comporta este doble aspecto de aquello que se perspectiviza y el acto de perspectivizar. Giere naturaliza la perspectiva, pero no al precio de confundir la donación (donatio) con los datos (data). En lugar de la acostumbrada oposición entre realismo y constructivismo, lo que tenemos es una oposición entre lo que es perspectivo y lo que no lo es ya que toda perspectiva nos obligará al doble esfuerzo de identificar y analizar la vertebración mutua de naturaleza y construcción. Nunca se puede abandonar la perspectiva propia. Por lo que acabamos de apuntar, el autor modifica el realismo constructivo por un perspectivismo científico. Como constata Michela Massimi, el

\footnotetext{
30 Ibíd.
} 
perspectivismo científico es una via media saludable (healthy via media) contra la rivalidad y oposición de los dos grandes bloques (realista y constructivista):

Ron Giere ha luchado por una versión del perspectivismo científico como una saludable via media entre lo que él Ilama "realismo objetivo", por un lado -es decir, la visión de que la ciencia nos concede un conocimiento de la naturaleza del ojo de Dios [God's eye knowledge of nature]- y las trampas de un relativismo alocado, por el otro lado ${ }^{31}$.

La via media gieriana se enemista con una concepción de la ciencia con atribuciones típicas de la omnisciencia divina, así como con un relativismo que antepone la perspectiva individual de la realidad natural a lo común de la misma que es compartido por la pluralidad de sujetos. Es Giere y no nosotros quien tiene una fijación en el relativismo, entendiéndolo como una perversión o desviación de la misma perspectiva humana. Para Giere, hay elementos que se muestran como comunes a toda perspectiva (universalidad), toda vez que cada perspectiva humana es privada e individual (parcialidad). Si sacamos partido de la anterior metáfora de Ortega, cada perspectiva es como un haz irrepetible e inigualable en su luminosidad de la única luz de la realidad, pero esta luz, aunque se multiplica según la circunstancia de cada sujeto, tiene una disposición que está presente en cada uno de los halos, es decir, en la perspectiva de cada cual. El ordenamiento natural e inteligible fundamental de la realidad no se pierde en cada una de las perspectivas, lo cual avala la posibilidad de una ciencia perspectivizada pero que es ciencia universal, a fin de cuentas.

En la neurofenomenología, más de una década antes de Giere, Francisco Varela ya quería establecer una vía media entre la imagen de la ciencia neutral y la de la ciencia construida, entre las ciencias cognitivas de la inmersión del ser humano en el mundo y aquellas que destacan sus funciones cognitivas superiores. Varela ambicionó que la ciencia tuviera en cuenta como doble aspecto de su tarea, pero una única perspectiva el lado subjetual y el lado objetual, de modo que esto nos permita

buscar una vía media entre el Escila de la cognición como recuperación de un mundo

31 Massimi, Michela. "Scientific perspectivism and its foes", Philosophica, No LXXXIV, 2012, p. 26. 
externo pre-dado (realismo) y el Caribdis de la cognición como proyección de un mundo interno pre-dado (idealismo). Ambos extremos se basan en el concepto central de representación: en el primer caso la representación se usa para recobrar lo externo; en el segundo se usa para proyectar lo interno ${ }^{32}$.

El concepto de representación hace las veces de estorbo ya que nos hace olvidar la capacidad previa representadora, es decir, desconoce que la perspectiva no es ni sólo lo representado (lo externo-objetivo) ni la potestad representadora (lo interno-subjetual). Varela compara esto, como también lo comparará Giere, con el tema del color: "Hemos visto que los colores no están 'ahí afuera', al margen de nuestra aptitud perceptiva y cognitiva. También hemos visto que los colores no están 'aquí dentro', al margen de nuestro entorno biológico y cultural. Contrariamente a la perspectiva objetivista, las categorías de color son experienciales; contrariamente a la perspectiva subjetivista, las categorías de color pertenecen a nuestro mundo biológico y cultural compartido. Giere asiente en esto: "las perspectivas del color son intersubjetivamente objetivas"33. El estudio del color nos permite apreciar la obvia afirmación de que la gallina y el huevo, el mundo y quien lo percibe, se definen recíprocamente"34.

El caso del color le sirve a Varela para destacar la peculiaridad multifactorial de la perspectiva, la cual consta de experiencia (la cual tiene además y no solamente representación) y de unas raíces en la vida tanto físico-biológica como culturalizada. Y es que, sin unos tonos de luz objetivos, sin los fotoreceptores de los órganos visuales dentro del sistema nervioso, sin un tratamiento cognitivo e interpretativo del yo consciente, no habría propiamente perspectiva del color. Aquí se ve el amplio espectro de la perspectiva y la multiplicidad de elementos que intervienen en ella. No se trata, para nada, de buscar el color fuera (como hechos ya dados en el mundo sin la percepción) o en la sola invención subjetivista: en color se da en la perspectiva que se apoya en todos los elementos anteriores.

La perspectiva en Varela aúna todos esos factores diversos gracias a que está radicada en la acción corporalizada (enacción) del ser humano, lo cual sugiere,

32 Varela, Francisco J.; THOMPSON, Evan Y Rosch, Eleanor, De cuerpo presente, Barcelona: Gedisa, 1997, p. 202.

${ }_{33}$ Giere, Ronald N., Scientific perspectivism, op. cit., p. 14.

${ }^{34}$ Varela, Francisco J. et. al. De cuerpo presente, op. cit., p. 202. 
"primero, que la cognición depende de las experiencias originadas en la posesión de un cuerpo con diversas aptitudes sensorio-motrices; segundo; que estas aptitudes sensorio-motrices están encastradas en un contexto biológico, psicológico y cultural más amplio"35. Por esto, Varela fundó el movimiento enactivista y entendió por "enacción" que los procesos sensorio-motores y la percepción han de ir unidos en la cognición humana; sin olvidar que han evolucionado juntos.

Como Giere, la neurofenomenología de Varela revindicaba la necesidad de profundizar en la metodología de las neurociencias como remedio anti-relativista y anti-dogmatista, incidiendo en especial en un método sistémico donde cupiesen desde su especificidad, pero también desde su unidad en la misma naturaleza tanto el método científico objetivo externo como el fenomenológico objetivo. Escribía, entonces, el neurobiólogo que no debería haber difidencia entre ambos sino una confianza mutua (mutual trust) entre ambos porque la perspectiva o experiencia los amista a los dos: "Lo que se necesita es un método estricto y ahí es donde radica tanto la dificultad como el potencial revolucionario (revolutionary potential) del tema"36.

\section{LA NEUROFENOMENOLOGÍA: UN TIPO DE PERSPECTIVISMO CIENTÍFICO}

\subsection{La perspectiva de partida: la experiencia vivida}

Por lo que llevamos escrito hasta ahora, se podría decir sin demasiados problemas que la neurofenomenología es un tipo de perspectivismo científico, si bien más fundamental todavía que el gieriano. Pero podríamos agregar más datos a esta idea. Varela abogó por un viraje revolucionario en la metodología de las ciencias, que sin dejar de lado la nota de poder referirnos al mundo sin dudas pirrónicas, devolviese su lugar al punto de vista de la primera persona, el cual se desarrolla como actividad de la conciencia:

De ahí que nuestro interés por la conciencia se especifique alrededor de la investigación de nuestra actividad consciente, siempre que ella se percibe a sí misma en el

${ }^{35}$ Ibíd., p. 203.

${ }^{36}$ Varela, Francisco J., "Neurophenomenology: A methodological remedy for the hard problem", Journal of Consciousness Studies, No III, 4, 1996, p. 335. 
momento de desarrollare en un modo operativo e inmanente y que es a la vez habitual y preflexivo ${ }^{37}$.

Para Varela, discutir de conciencia, más que de una substancia, es discutir de experiencia, esto es, de una actividad; una actividad inmanente y natural al hombre. No es una experiencia sublimada o espiritualista sino una experiencia vivida ("lived experience"38). En lugar de usar reiteradamente la palabra "perspectiva", el neurobiólogo se sirve del término "experiencia" (experience), el cual se relaciona más estrechamente con su formación científica. Leemos en su programa sobre la neurofenomenología que "debemos examinar, más allá del fantasma de la subjetividad, las posibilidades de concretas de un examen de la experiencia que está en el corazón mismo de la inspiración fenomenológica"39. Giere también se quejaba del tratamiento idealista mediante el cual las estados y eventos mentales "son retratados como siendo puramente experiencias subjetivas" 40. No es, pues, un subjetivismo espectral o idealista el movimiento que funda Varela y que, con matices, inspira a Giere, sino el examen riguroso de la experiencia, una experiencia que no es únicamente autorreferencial. La neurofenomenología está pensada para integrar "la experiencia subjetiva y la dinámica cerebral en la neurociencia de la conciencia"41.

Pero, mutatis mutandis, la experiencia que la neurofenomenología pretende salvar no sólo remite al mundo, sino que es mundo en su cualidad perspectiva. Con esto queremos señalar que esta experiencia no es fruto de un estilo empirista de pensar puesto que todo dato del mundo es inaccesible sin la experiencia consciente donante. Los data de las ciencias empíricas no tendrían ni sentido ni entidad para nadie a no ser por una experiencia, por una perspectiva donans, es decir donadora primordial de sentido. Veamos este perspectivismo matriz que Varela le debe especialmente a Edmund Husserl y que lo hace más cardinal aún la aplicación de protocolos neurocientíficos.

\footnotetext{
${ }^{37}$ Depraz, Natalie; Varela, Francisco J. y Vermersch, Pierre, À l'épreuve de l'expérience. Pour une pratique phénoménologique, Bucarest: Zeta Books, 2011, pp. 13-14.

${ }^{38}$ Varela, Francisco J. Y Shear, Jonathan, The view from within, Ohio: Imprint Academic, 1999, p. 1.

39 Varela, Francisco J., "Neurophenomenology", op. cit., p. 335.

${ }^{40}$ Giere, Ronald N., Scientific perspectivism, op. cit., p. 37.

41 Thompson, EVAN Y LUTZ, Antoine. "Neurophenomenology. Integrating Subjective Experience and Brain Dynamics in the Neuroscience of Consciousness", Journal of Consciousness Studies, No XX, 9-10, 2003 , p. 32.
} 


\subsection{La fenomenología de Husserl como "perspectivismo matriz"}

Para Francisco Varela, Husserl fue quien con más fuerza privilegió la perspectiva, en especial como la modalidad de conciencia por la que el sujeto tiene y explicita para sí no sólo las estructuras y procesos mentales sino las cosas del mundo. En este sentido, Husserl afirmó "haber descubierto por este proceso un nuevo ámbito (domaine) previo (préalable) a toda ciencia empírica"42. Años más tarde, Giere compartirá la misma crítica de Varela en el sentido de que en las ciencias naturalizadas de forma reduccionista se "implica el rechazo de apelaciones a reclamos a priori de cualquier tipo"43.

Pero no debemos desorientarnos: constatar que el nuevo dominio de la perspectiva consciente sea previo no lo es al modo de una región independiente a toda res objetiva, sino que es previo en el orden ontológico: de hecho la misma perspectiva (Varela la denomina "experiencia") confirma que lo más inmediato, directo e indudable es la facultad consciente que permite la perspectiva y sus objetos, sin la cual no habría experiencia de nada. Alrededor de 1908, Edmund Husserl, con anterioridad a la primera parte de sus Ideas relativas a una filosofía pura y una filosofía fenomenológica, había asignado a su filosofía el apelativo de "idealismo trascendental", sin desistir de él incluso en sus obras de madurez. Debemos acudir a las Conferencias de París de 1929 para que Husserl nos aclare al menos qué no quiere decir con esta expresión.

Allí el pensador nos revela, ante todo, lo que es el idealismo trascendental, es decir, "que el ego, para decirlo con los antiguos antecesores, tiene un inmenso a priori innato y que toda la fenomenología, o el autoexamen puro del filósofo conducido metódicamente, es descubrimiento de este a-priori innato en su infinita multiformidad"44. El idealismo trascendental tiene como como consistencia y finalidad "hacer accesible sistemáticamente la esfera trascendental como la esfera absoluta del ser y de la constitución, a la cual está referido todo lo concebible"45. Hay que llegar a lo trascendental ya que como sugiere Giere que hay,

42 Varela, Francisco J.; THOMPSON, EVAN y ROSCH, ELEANOR, L'inscription corporelle de l'esprit, Paris: Seuil, 1993, p. 44.

${ }_{43}$ Giere, Ronald N., Scientific perspectivism, op. cit., p. 11.

${ }^{44}$ Husserl, Edmund, Las conferencias de París. Introducción a la fenomenología trascendental, México: Instituto de Investigaciones Filosóficas (UNAM), 1988, p. 38.

${ }^{45}$ Ibíd., p. 39. 
desde principios del siglo $\mathrm{XX}$, "una dramática presentación de la parcialidad de las perspectivas"46. Husserl en lo trascendental, como Varela en lo experiencial, se empecinó en llegar a la unidad primera de esta diversidad perspectivística.

En la IV ponencia sostenía Husserl que su idealismo trascendental "no es un idealismo psicológico, no un idealismo que a partir de datos sensibles carentes de sentidos quiera derivar un mundo pleno de sentido; no un idealismo kantiano, que por lo menos como concepto límite cree poder dejar abierta la posibilidad de un mundo de cosas en sí"47. Ciertamente, toda realidad en sí viene mediada inexorablemente por el para-mí perspectivo, pero no como posible contra Kant sino como actual. Esta postura es muy semejante al "realismo constructivo" del Giere de los años 90 y que antecede al constructivismo en ciencia. $Y$ es que contamos con una locuaz afirmación de Husserl que confirma nuestra interpretación:

Un mundo real pertenece necesariamente como correlato intencional a un yo real, a su real corriente de conciencia. Un mundo es lo que es sólo como aquello que está frente a un yo experienciante; todo lo que (el mundo) contiene, es o bien directamente experienciado o pertenece a un horizonte de indeterminación determinable de lo actualmente experienciable ${ }^{48}$.

Los fenómenos del yo son manifestaciones (Anzeichen), actuales en la perspectiva humana, de la realidad del mundo. La realidad viene a habitar en la perspectiva, si bien no como éxito insuperable sino como conquista todavía por conseguir. Por ello bien se ha dicho de Husserl que mantuvo un "trascendentalismo metódico"49, en parte similar al naturalismo metodológico de Giere, por el que la perspectivización de los objetos del mundo no es completa (como pasaba, por ejemplo, en un Hegel en el que todo lo real era de antemano espíritu), sino abierta a un mejor y perfectible acceso al mundo. Si el naturalismo metodológico de Giere enfatiza la perfectibilidad de la ciencia mediante el perfeccionamiento de sus metodologías, el trascendentalismo metodológico de Husserl enfatiza la

${ }^{46}$ Giere, Ronald N., Scientific perspectivism, op. cit., p. 35.

47 Husserl, Edmund., Las conferencias, op. cit., p. 45.

48 Husserl, Edmund., Transzendentaler Idealismus, Hua., XXXVI, p. 121, en J. C. Vargas Bejarano, "El proyecto de una filosofía trascendental no idealista", Estudios filosóficos, No XLVII, 2013, p. 51.

${ }^{49} \mathrm{Cfr}$. Tengelyi, Laszlo, "Der methodologische Transzendentalismus der Phänomenologie", en C. Ierna et. al., eds., Philosophy, phenomenology, sciences. Essays in commemoration of Edmund Husserl, Dordrecht: Springer, 2010. 
perfectibilidad del conocimiento en cuanto tal sobre la base del perfeccionamiento de la fenomenología como una disciplina que llegue a estudiar el campo perspectivo, sus contenidos y modalidades. Giere sostiene que su naturalismo metodológico "apoya la investigación científica tal y como ciertamente han trazado los mejores medios humanos para entender tanto el mundo natural como a estos mismos como parte del mundo"50. Es decir, apoya a la ciencia toda vez que resitúa como parte de un mundo provisto de una perspectiva prevaleciente. Incluso Giere ha "desarrollado la conexión entre naturalismo y pragmatismo"51.

Más el trascendentalismo metódico de Husserl que inspira a Varela es todavía más audaz y ambicioso que el naturalismo metodológico gieriano. Su fenomenología, que está en la base de Varela, se nos muestra cómo un perspectivismo más perentorio que el científico de Giere porque no sólo estudia el conocimiento aplicado de la ciencia sino el conocimiento en sí mismo, o aún más, su posibilitación constitutiva presupuesta ya en las diversas perspectivas de la ciencia. La fenomenología se erige como la teoría ya no de la simple aprehensión sino de cualquier acto de la perspectiva. Por eso, Husserl defendió que la tarea más inaplazable consistía en ocuparse de esta posibilitación común del conocimiento de cualquier ciencia u ontología regional, es decir, consagró la fenomenología como philosophia prima.

Podríamos tildar a la fenomenología husserliana, en la renovación a la que nos dirige Varela, como un "perspectivismo matriz" ("experiencialismo matriz", para ser consecuentes con su vocabulario) en el que la ciencia empírica, o cualquier ciencia, podría nacer como su vástago o perspectiva aplicada: una vez que sabemos algo de los entresijos del conocimiento primero, tendremos menos dificultades para obtener los conocimientos particulares que se radican en él. Es, pues, más urgente la fenomenología que cualquier otra empresa porque, adelantándose a la bifurcación de la perspectiva en una cosmovisión realista o empirista del mundo, le preocupa ante todo saber algo de la perspectiva per se, en esencia (Wesen), no tanto por lo que aporta sino por su cualidad constitutiva de perspectivizar. La fenomenología, entonces, se sumerge en el interior de la perspectiva (de cualquier perspectiva) y rescata las condiciones trascendentales y a-

${ }^{50}$ Giere, Ronald N., Scientific perspectivism, op. cit., p. 16.

${ }^{51}$ Ibíd., p. 120. 
temáticas del conocimiento trascendente y temático, sea éste filosófico o científico.

\subsection{Actitud natural y neurociencias cognitivas: un enactivismo perspectivista}

Varela se percató de que la fenomenología verdaderamente se oponía a un realismo reo de ingenuidad, como lo llamaba Giere, gracias a la puesta en suspense de este tipo de posturas dogmáticas con la epojé. Y es que Husserl, nos confiesa el neurobiólogo, "requería poner aparte, como entre paréntesis, los juicios vulgares acerca de la relación entre la experiencia y el mundo", es decir, "llamó 'actitud natural' al punto de vista desde el cual se realizan estos juicios vulgares; es la actitud generalmente denominada 'realismo ingenuo', que consiste en la convicción de que el mundo es independiente de la mente o la cognición y de que las cosas generalmente son tal como aparecen"52.

En la actitud natural, si empleamos el lenguaje husserliano ${ }^{53}$, que pretende controlar solamente los hechos objetivos del mundo en su existencia exterior, la ciencia empírica proscribió a un total descontrol a la subjetividad, con el riesgo que comporta creer ingenuamente en una transparencia plena y diáfana (sinónimo de una falsa inoperancia del sujeto) en la cual nos llegarían pasiblemente los hechos del mundo. Pese a todo, la actitud natural no debe ser confundida en Husserl: no consiste sin más en el estudio de la naturaleza que acomete la ciencia empírica. Si así fuera, Husserl, de formación científica, hubiese sido un enemigo de la ciencia. "Actitud natural", pues, no es otra cosa que considerar los hechos del mundo como al margen de la consideración o influencia de la mente y, en el caso más extremo, como si esta fuera el único camino científico de saber algo sobre el mundo. Varela suscribió una interpretación de Husserl como un naturalista no-reductivista, es decir, comprendiendo a la naturaleza no como simple concentración de átomos sino como una naturaleza amplia y compleja, en la cual encuentran morada las formas cada vez más perfectas de vida consciente.

Por eso, Varela creyó necesario desembarazarnos de malentendidos, sobre todo de aquellos que nos llevarían a interpretar a Husserl como un enemigo de la investigación objetiva de la naturaleza: se puede afirmar que Husserl fue un

52 Varela, Francisco J. et. al., De cuerpo presente, op. cit., p. 40.
53 Cfr. Husserl, Edmund., La filosofía, ciencia rigurosa, Madrid: Ediciones Encuentro, 2009. 
enemigo del realismo metafísico e ingenuo pero no un enemigo de la objetivación, de la cual encuentra su razón a través de su método fenomenológico. De aquí se sigue que la neurofenomenología vareliana fuese una forma peculiar de perspectivismo ni aplicado a lo que hoy se ha profesionalizado con el nombre de "neurociencias cognitivas". En este llamativo enactivismo perspectivista, Varela busca integrar, en una misma perspectiva metodológica y gracias a un enfoque enactivo, tanto los aportes empíricos de las neurociencias como los aportes subjetuales de la fenomenología, aunque gozando estos últimos de una preponderancia perceptual con respecto de los anteriores:

El enfoque enactivo de la percepción no procura determinar cómo se recobra un mundo independiente del perceptor, sino determinar los principios comunes de ligamiento legal entre los sistemas sensoriales y motores que explican cómo la acción puede ser guiada perceptivamente en un mundo dependiente del perceptor ${ }^{54}$.

En neurofenomenología enactiva, el perceptor es relevante no solamente como cuerpo que tiene experiencia, o también fracturas o lesiones (por ejemplo), sino que recobra su importancia al interior mismo de su misma perspectiva. En sintonía con Varela, la propuesta de Giere clama también por ser "más radicalmente perspectiva que la mayor observación instrumental"55, esto es, que la simple instrumentalización y descripción de las cosas como independientes de quien perspectiviza tanto sensorial como motoramente. No hay acción consciente desencajada del cuerpo sino un quién con su propio punto de vista corporalizado. Sobre este tema, hemos descubierto que Giere menciona expresamente a De cuerpo presente (The Embodied Mind, en inglés) de Varela, reconociendo que está "entre las más tempranas e influyentes obras" a favor de la idea, que Giere suscribe abiertamente, de que "la cognición está corporalizada"56. Esta corporalización no sólo no se opone, sino que abre la vía a la neurociencia. Al igual que el neurobiólogo, este autor reconoce que, por ejemplo, "la neurociencia de la visión del color es actualmente un área muy activa de investigación"57.

\footnotetext{
${ }^{54}$ Varela, Francisco J. et. Al., De cuerpo presente, op. cit., p. 203.

55 GIERE, Ronald N., Scientific perspectivism, op. cit., p. 41.

56 Íbid., p. 114.

${ }^{57}$ Varela, Francisco J. et. al., De cuerpo presente, op. cit., p. 32.
} 
En el enactivismo vareliano, la perspectiva no está solamente en función de lo perspectivizado sino de esa la acción corporalizada del hombre total, siendo decididamente más radical que Giere. Así pues, la percepción es una dimensión destacable, aunque no la única, de la perspectiva matriz de la fenomenología husserliana. En la perspectiva corporalizada, la cual incluye en su enacción a la percepción, se ligan las funciones sensorio-motoras, así como las funciones cognitivas de una vida tanto biológico como socio-histórica. Varela, al igual que Giere, pero precediéndolo cronológica y temáticamente, consideraba algo fuera de lugar, racionalmente hablando, la voluntad de querer negar o poner en entredicho tal perspectiva por parte de cualquier docto naturalista puesto que un "yo consiste en tener un 'punto de vista' coherente y unificado, una perspectiva estable y constante desde la cual pensamos, percibimos y actuamos"58. La perspectiva, pues, es innegable para el neurocientífico y para cualquier científico que se precie, si bien la novedad de la neurofenomenología no se queda simplemente en teorizar globalmente acerca de un perspectivismo científico sino en corporalizarlo radicalmente, así como en llevarlo y aplicarlo en las neurociencias cognitivas.

De hecho, el neurobiólogo consideraba que "la sensación de que tenemos (¿somos?) un yo parece tan incontrovertible que nos parece absurdo que alguien - aunque sea un científico- la cuestione o la niegue"59. Varela vio, pues, en la neurofenomenología

un radical viraje paradigmático [...], la convicción de que las verdaderas unidades de conocimiento son de naturaleza eminentemente concreta, incorporadas, encarnadas, vividas; que el conocimiento se refiere a una situacionalidad [...]. Lo concreto no es un paso hacia otra cosa, [...], es cómo llegamos y cómo permanecemos ${ }^{60}$.

Los métodos de las neurociencias aportarán los datos en tercera persona de esta situacionalidad, datos ininteligibles sin la perspectiva o experiencia primordial que permite su tratamiento y comprensión como un todo de sentido. La neurofenomenología toma este punto de vista de la tercera persona siempre desde

\footnotetext{
${ }^{58}$ Ibíd., 76.

59 Ibíd.

60 Bedia, Manuel Y Castillo, Luis Fernando., "Hacia una teoría de la mente corporizada: la influencia de los mecanismos sensomotores en el desarrollo de la cognición", Ánfora, No XXVIII, 2010, p. 111.
} 
el ángulo de la primera persona que tiene una importancia trascendental, en la línea husserliana, pero con un interés neurobiológico totalmente novedoso:

Mientras que los enfoques estándar a los correlatos neuronales de la conciencia han asumido una relación explicativa unidireccional entre los sistemas neuronales neuronales-internos representacionales y los contenidos de conciencia, nuestro enfoque permite teorías e hipótesis sobre la relación recíproca y bidireccional entre los estados conscientes y la actividad neuronal local61.

En vez de esta relación unidireccional, la neurofenomenología, debido a su compromiso por una corporalización radical (radical embodiment), se afana en estudiar la perspectiva humana (la experiencia vivida) en su aspecto bidireccional ( neurofisiológico en tercera persona y fenomenológico en primera persona) mediante el entrenamiento (en segunda persona) de sujetos preparados. La neurofenomenología, pues, "defiende la importancia de la recopilación de datos en primera persona por parte de sujetos entrenados fenomenológicamente como una estrategia heurística para describir y cuantificar los procesos fisiológicos relevantes para la conciencia"62.

\section{CONCLUSIONES}

1) La neurofenomenología se inscribe en la tradición perspectivista occidental que comenzó en la Modernidad y ha continuado hasta nuestros días. La diferencia estriba fundamentalmente en que el perspectivismo de Giere y la neurofenomenología de Varela avanzan de la mera especulación filosófica de sus predecesores sobre la perspectiva al campo de la ciencia e incluso empirizándola, como hemos visto, mediante el caso concreto de la experiencia del color.

2) El perspectivismo científico de Ronald N. Giere se sitúa en la tradición científica post-empirista (desde Heisenberg) y tiene la novedad, con respecto a Nietzsche u Ortega, de llevar este enfoque al campo aplicado de la filosofía de la ciencia. Los autores anteriores a Giere no han sistematizado la cuestión del perspectivismo ni en la ciencia ni en su filosofía aplicada. Incluso ha afirmado Giere

${ }^{61}$ Varela, Francisco J. Y Thompson, E., "Radical embodiment: neural dynamics and consciousness", Trends in Cognitive Science, No V, 10, 2001, p. 418.

62 Thompson, Evan Y Lutz, Antoine., "Neurophenomenology", op. cit., p. 32. 
que su enfoque es útil para pulir y perfeccionar el método científico, el cual no sólo está llamado a descubrir objetos sino, máximamente, a revisar y optimizar sus presupuestos (teorías, modelos, leyes, intereses, etc.). Giere quiso encontrar un equilibrio entre un constructivismo radical y un realismo metafísico, repudiando en uno y otro caso el relativismo. Es mejor hablar de perspectivismo y no de relativismo porque no se incurre en afirmaciones sobre el ser de lo real ni sobre la posibilidad o imposibilidad taxativas de conocerlo.

3) La neurofenomenología de Francisco J. Varela precede tanto en la cronología como en la temática a Giere siendo todavía más radical que la suya. No se contenta con referirse a la ciencia in abstracto, sino que aterriza su reflexión en un campo definido de la ciencia (la neurociencia cognitiva). Nos hallamos, pues, ante una estrategia heurística, es decir, un recurso organizativo teórico-práctico que persigue poner en relación con la experiencia (perspectiva) con el mundo y los procesos neurofisiológicos que la arbitran, mediante el entrenamiento de los sujetos en segunda persona. La neurofenomenología, pues, da una mayor explicatividad a esta experiencia (perspectiva).

4) Esto tiene en común con Giere en el hecho de que Varela también trabaja en el campo científico, aunque pretende armonizar el punto de vista subjetivo con el objetivo en los protocolos neurocientíficos, sin renunciar a uno en favor del otro. Giere y Varela se encaminan en una vía media entre los extremismos del polo subjetivo y el polo objetivo de la experiencia. De hecho y como hemos descubierto, Giere cita a Varela y le presta su reconocimiento. Así mismo, hay similitudes tanto en Varela como Giere en criticar el naturalismo y en declararse, sin reduccionismos y tan sólo metodológicamente, naturalistas. Entre las diferencias, encontramos que Varela introduce un original enactivismo perspectivista, aunque no usa técnicamente el término "perspectiva" sino "experiencia". Giere, por contra, incluye la experiencia (del color, por ejemplo) dentro de la perspectiva.

Además, en lugar solamente de la perspectiva asociada en lo inmediato a sus estados y contenidos, a Varela le atrajo hondamente el carácter matriz de la experiencia. He aquí una influencia husserliana que no notamos para nada en el otro autor y que le guía hacia un propio perspectivismo o experiencialismo matriz en la raíz trascendental de las contrapartes corporalizadas de la experiencia. En este sentido, el neurobiólogo demostró un afianzamiento en la cualidad 
trascendental de la experiencia que no hallamos, debido a la disparidad de tradiciones mutuas, en Ronald N. Giere. Varela, al defender un entrenamiento de los sujetos que participan en sus protocologs neurfenomenológicos defiende a la vez la integración de los puntos de vista de la primera, la segunda y la tercera personas.

\section{BIBLIOGRAFÍA}

BediA, Manuel y Castillo, Luis Fernando. "Hacia una teoría de la mente corporizada: la influencia de los mecanismos sensomotores en el desarrollo de la cognición". Ánfora, núm. 2010, pp. 101-124.

CAPRA, Fritjof. La trama de la vida, Barcelona: Anagrama, 1996.

Depraz, Natalie; Varela, Francisco J. y Vermersch, Pierre. À l'épreuve de l'expérience. Pour une pratique phénoménologique. Bucarest: Zeta Books, 2011.

GIERE, Ronald N. Science without laws. Chicago: The University of Chicago Press, 1999.

GIERE, Ronald N. Scientific perspectivism. Chicago: The University of Chicago Press, 2006.

HUSSERL, Edmund. La filosofía, ciencia rigurosa. Madrid: Ediciones Encuentro, 2009.

-, Introducción a la fenomenología trascendental. México: Instituto de Investigaciones Filosóficas, UNAM, 1988.

-, Transzendentaler Idealismus. En Vargas Bejarano, J. C. "El proyecto de una filosofía trascendental no idealista". Estudios filosóficos, núm. 47, 2013, pp. 35-57.

LACHELIER, Henri. "Introduction". En LeIBNIZ, G. W. Nouveaux Essais sur I'Entendement Humain. París: Librarie Hachette, 1898, pp. 1-10.

LeIBNIZ, Gottfried Wilhelm. Oeuvres. Vol. 1. Nouveaux Essais sur I'Entendement. Opuscules divers. Lyon: Charpentier, 1842.

Massimi, Michela. "Scientific perspectivism and its foes". Philosophica, núm. 84, 2012, pp. 25-52. 
MORRISON, Margaret. "One phenomenon, many models: Inconsistency and complementarity". Studies in History and Philosophy of Science, no 42, vol. 2, 2011, pp. 342-351.

NicolÁs, Juan Antonio. "Gnoseología y perspectivismo corporal en Leibniz". Cuadernos Salmantinos de Filosofía, núm. 5, vol. 10, 2001, pp. 135-150. NIETZSCHE, Friedrich. Jenseits von Gut und Böse. Vorspiel einer Philosophie der Zukunft. En De Santiago Guervós, L. "Las ilusiones del conocimiento: perspectivismo e interpretación". Thémata, núm. 27, 2001, pp. 123-139.

-, Menschliches, Allzumenschliches. En De Santiago Guervós, L. "Las ilusiones del conocimiento: perspectivismo e interpretación". Thémata, núm. 27, 2001, pp. 123-139.

ORTEGA y GASSET, José. Obras Completas. Vol. 2. "Verdad y perspectiva". Madrid: Taurus, 2004, pp. 159-164.

Putnam, Hilary. Reason, Truth, History. Cambridge: Cambridge University Press, 1981.

RUeger, Alexander. "Perspectival models and theory unification". British Journal for the Philosophy of Science, núm. 56, vol. 3, 2005, pp. 579-594.

SÁNCHEZ RodrígueZ, Manuel. "El perspectivismo en los Nuevos ensayos sobre el entendimiento humano de Leibniz". Dissertatio: Revista de Filosofía, vol. compl. 3, 2016, pp. 86-108.

TENGELYI, Laszlo. "Der methodologische Transzendentalismus der Phänomenologie". En Ierna, C.; Jaccobs, H. y Mattens, P. (Eds.). Philosophy, phenomenology, sciences. Essays in commemoration of Edmund Husserl. Dordrecht: Springer, 2010, pp. 135-153.

THOMPSON, Evan y LUTZ, Antoine. "Neurophenomenology. Integrating Subjective Experience and Brain Dynamics in the Neuroscience of Consciousness". Journal of Consciousness Studies, núm. 10, vol. 9-10, 2003, pp. 31-52.

VAN FRASSEN, Bas. Scientific representation: Paradoxes of perspective. Nueva York: OUP, 2008.

VARELA, Francisco J. "Neurophenomenology: A methodological remedy for the hard problem". Journal of Consciousness Studies, núm. 3, vol. 4, 1996, pp. 330-349.

-, De cuerpo presente. Barcelona: Gedisa, 1997.

VARELA, Francisco J. y SHEAR, Jonathan. The view from within. Ohio: Imprint Academic, 1999. 
VARELA, Francisco J. Y ThOMPSON, Evan. "Radical embodiment: neural dynamics and consciousness". Trends in Cognitive Science, núm. 5, vol. 10, 2001, 418-425.

VARELA, Francisco J.; ThOMPSON, Evan y RosCH, Eleanor. L'inscription corporelle de l'esprit. París: Seuil, 1993. 